\title{
Circular Dichroic Studies on Marmelo Lactones and the Related $\gamma$-Lactones with Unsaturation at the C-5 Position
}

\author{
Yoshihiro Nishida, Hiroshí Ohrui, Hiroshi Meguro \\ and Kenji MorI* \\ Department of Food Chemistry, Faculty of Agriculture, Tohoku University, \\ Tsutsumidori-Amamiyamachi, Sendai 980, Japan \\ * Department of Agricultural Chemistry, Faculty of Agriculture, \\ University of Tokyo, Bunkyo-ku, Tokyo 113, Japan
}

Received March 19, 1985

\begin{abstract}
The intense $\mathrm{CD}$ bands of marmelo lactones and the related $\gamma$-lactones with unsaturation at the C-5 position were studied in terms of the possible interactions between the $n \rightarrow \pi^{*}$ transition of the carbonyl and the $\pi \rightarrow \pi^{*}$ transition of the double bond at C-5, together with the preferred conformations about the $\mathrm{C} 4-\mathrm{C} 5$ axis. The $\mathrm{C} 4-\mathrm{C} 5$ axis of marmelo lactones takes a preferred conformation which is characteristic for the acyclic allylic alcohols and seems to be an important factor for the $(4 S)-\gamma$-lactones with a double bond at the C-5 position to give a negative coupling between the two chromophores.
\end{abstract}

Various kinds of lactones have been found in nature, some of which are known as sugar lactones, steroid lactones and terpene lactones. Determining their absolute configurations is an important aspect for their biosynthetic or biological study. For the saturated lactones, their CD (circular dichroism) signs due to the $\mathrm{n} \rightarrow \pi^{*}$ transition of the carbonyl have been well correlated with their absolute configurations $^{1 \sim 3)}$ and/or ring chiralities. ${ }^{4,5)}$ However, for the unsaturated lactones, which have a UV chromophore near the carbonyl, the interaction of the $n \rightarrow \pi^{*}$ transition with other UV chromophores may cause strong Cotton effects which often present some difficulties in their configurational and conformational analyses.

In our preceding papers, ${ }^{6,7)}$ we determined the absolute configurations of marmelo lactones $\mathbf{1}$ and $\mathbf{2}$ through their stereoselective syntheses. These lactones have a conjugated diene chromophore at the C-5 position and are closely related to the Japanese Beetle's sex pheromone 5 (Fig. 1). However, the CD signs of the two lactones were opposite in our preliminary experiments, ${ }^{8)}$ although their con- figurations at the C-4 position were the same. The saturation of the C-5 double bond into the saturated lactones $\mathbf{8} \sim \mathbf{1 0}$ also significantly changed their CD signs and magnitude. These results initially led to proposing a wrong conclusion in the absolute configurations of marmelo lactones, which was later synthetically corrected. ${ }^{6,9)}$ In order to settle this problem, we attempted in a separate paper ${ }^{10)}$ the application of the benzoate chirality method ${ }^{11)}$ for these $\gamma$-lactones, after converting them into acyclic alcohols.

In this paper, we study the CD of marmelo lactones and the related $\gamma$-lactones without

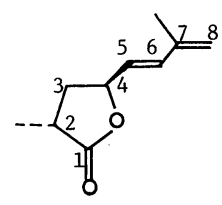

1

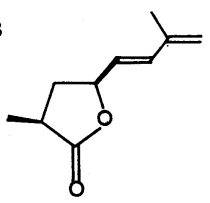

2

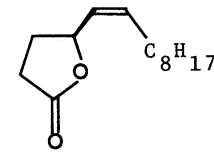

5
FIG. 1. Structures of (4S)-Marmelo Lactones 1 and 2, and $(4 S)$-Japanese Beetle's Sex Pheromone 5.

Numbering is referred to the conventional method for alkanoic acid lactones where the carbonyl carbon is numbered as $\mathrm{C}-1$. 
destruction of the lactone structure to rationalize their anomalous Cotton effects.

\section{MATERIALS AND METHODS}

a) Model compounds. The titled lactones $\mathbf{1}$ and 2, the related $\gamma$-lactones with various kinds of substituents at the C-4 position $(3 \sim 12)$, and the oxolanes $13 \sim 15$ were prepared from L-glutamic acid, ${ }^{6 \sim 8)}$ expect for the Japanese Beetle pheromone 5 which was prepared via the asymmetric reduction of an acetylenic keto ester. ${ }^{12)}$ Their syntheses and physical data have already been shown in our previous reports. ${ }^{6 \sim 8,12)}$

b) $C D, U V$ and NMR measurements. The CD spectra were measured on a Mark III-J Dichrograph at a concentration of about $0.01 \mathrm{mg} / \mathrm{ml}$. The UV were measured on a Hitachi Model 100 200 spectrometer at a concentration of about $1.0 \times 10^{-4} \mathrm{mg} / \mathrm{ml}$, and the ${ }^{1} \mathrm{H}-\mathrm{NMR}$ spectra, on a JEOL FX-100 spectrometer at about $4 \mathrm{mg} / \mathrm{ml}$. All the measurements were performed at $22 \sim 24^{\circ} \mathrm{C}$ and the solvents are cited in the text.

\section{RESULTS AND DISCUSSION}

a) Possible interaction between the lactone carbonyl and double bond at the $C-5$ position

The significant CD changes of marmelo lactones $\mathbf{1}$ and 2, and Japanese Beetle's sex pheromone 5 from their saturated lactones suggested a strong interaction between the lactone carbonyl and the C-5 double bond, which has not been discussed in the earlier works. In order to confirm this, we investigated the $\mathrm{CD}$ of the three types of compounds, $\mathbf{A}, \mathbf{B}$ and $\mathbf{C}$. Type $\mathbf{A}$ is the original of unsaturated lactones $\mathbf{1} \sim 7$,

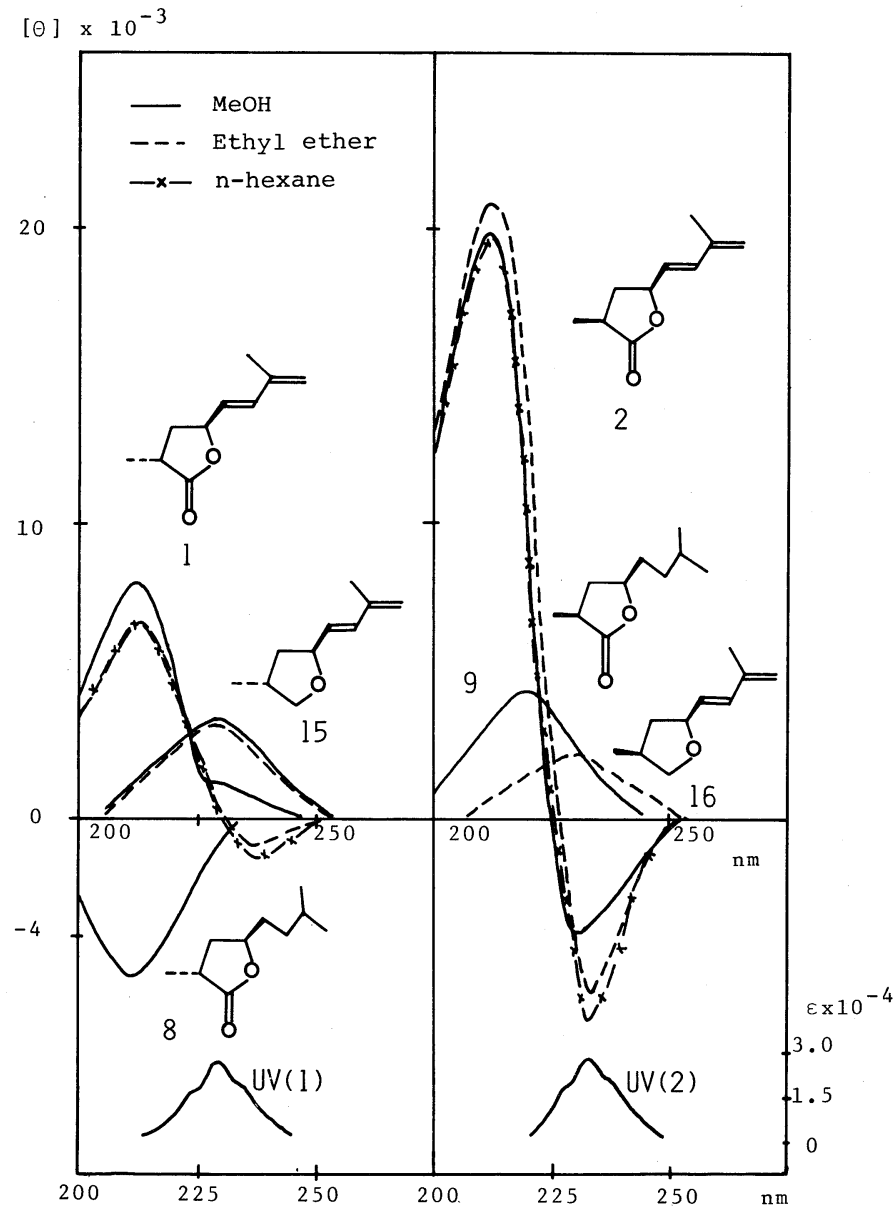

FIG. 2. CD and UV Spectra of Marmelo Lactones $\mathbf{1}$ and 2, Their Saturated Lactones $\mathbf{8}$ and 9, and Oxolane Derivatives 15 and 16. 


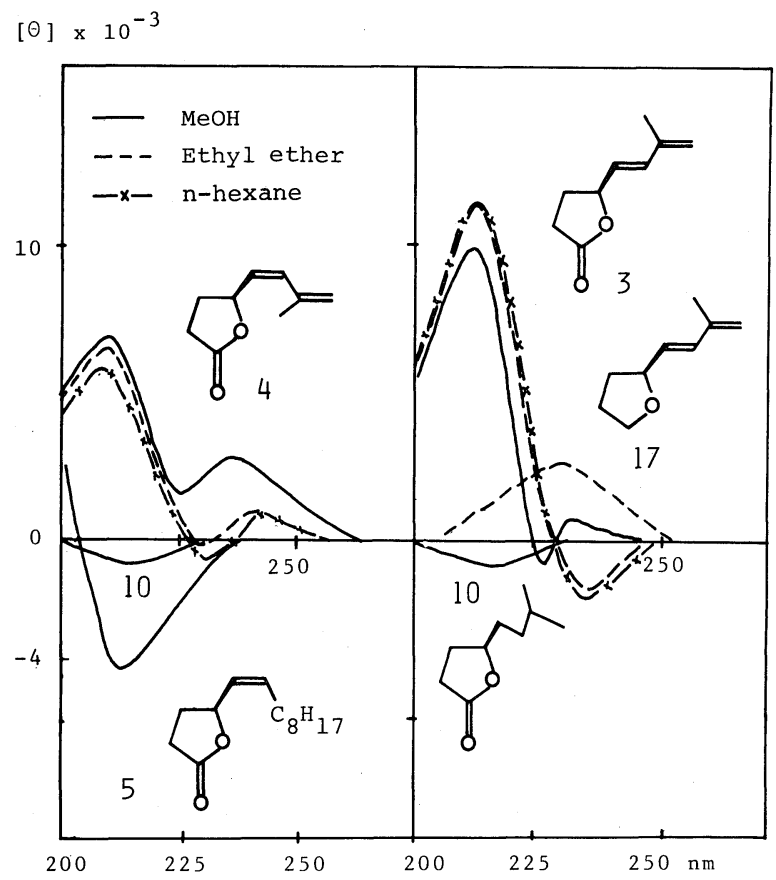

FIG. 3. CD Spectra of C-5 Unsaturated Lactones 3, 4 and 5, Their Saturated Derivative 10, and Oxolane 17.

and was further divided into three types: Type A-I is lactones with a conjugated diene at C-5, Type A-II is those with a non-conjugated double bond, and Type A-III is those with a carboxyl group in the side chain. Type $\mathbf{B}$ is saturated lactones free from the double bond chromophore of the Type A lactones at C-5, and Type $\mathbf{C}$ is oxolane derivatives free from the carbonyl chromophore of the Type $\mathbf{A}$ lactones.

The CD results of the Type $\mathbf{A} \sim \mathbf{C}$ compounds are shown in Table I and Figs. 2 and 3. The significant results can be summarized as follows:

1) The unsaturated lactones of Type A-I and A-III gave much more intense positive bands at $c a .215 \mathrm{~nm}$ than the saturated lactones of Type B.

2) The Type A-II lactone $\mathbf{5}$ also gave a much more intense $C D$ band than the saturated lactone 10; however, its negative sign was opposite to the intense bands of the Type A-I and A-III lactones with the same (4S)configuration.

3) The negative or ambiguous CD bands in the $c a .235 \mathrm{~nm}$ region of the Type A-I lactones changed to clear positive bands in a slightly shorter wavelength region $(229 \mathrm{~nm})$ for the Type $\mathbf{C}$ compounds.

Marmelo lactones and the related lactones of Type A-I gave two CD maxima in the $215 \mathrm{~nm}$ and $235 \mathrm{~nm}$ regions. The former band could be attributed to the $\mathrm{n} \longrightarrow \pi^{*}$ transition of the carbonyl, and the latter to the $\pi \longrightarrow \pi^{*}$ transition of the conjugated diene chromophore, judging from their UV spectra. Both the two bands of the Type A-I lactones showed significant differences in the sign and magnitude from those of the Type $\mathbf{B}$ and the Type $\mathbf{C}$ compounds. The changes indicate strong interactions between the $\mathrm{n} \longrightarrow \pi^{*}$ transition and the $\pi \rightarrow \pi^{*}$ transition for the Type A-I lactones, to which intense CD bands in the $c a .215 \mathrm{~nm}$ region could be attributed.

Similar interactions have been already noticed for the $\beta, \gamma$-unsaturated ketones, which gave an intense $\mathrm{n} \longrightarrow \pi^{*}$ band of the carbonyl in the $c a .290 \mathrm{~nm}$ region. In recent theoretical studies on the $\beta, \gamma$-unsaturated ketones, ${ }^{13 \sim 15)}$ this interaction has been explained by elec- 
Table I. Chiroptical Data of $\gamma$-Lactones with Unsaturation at C-5, and the Related Compounds

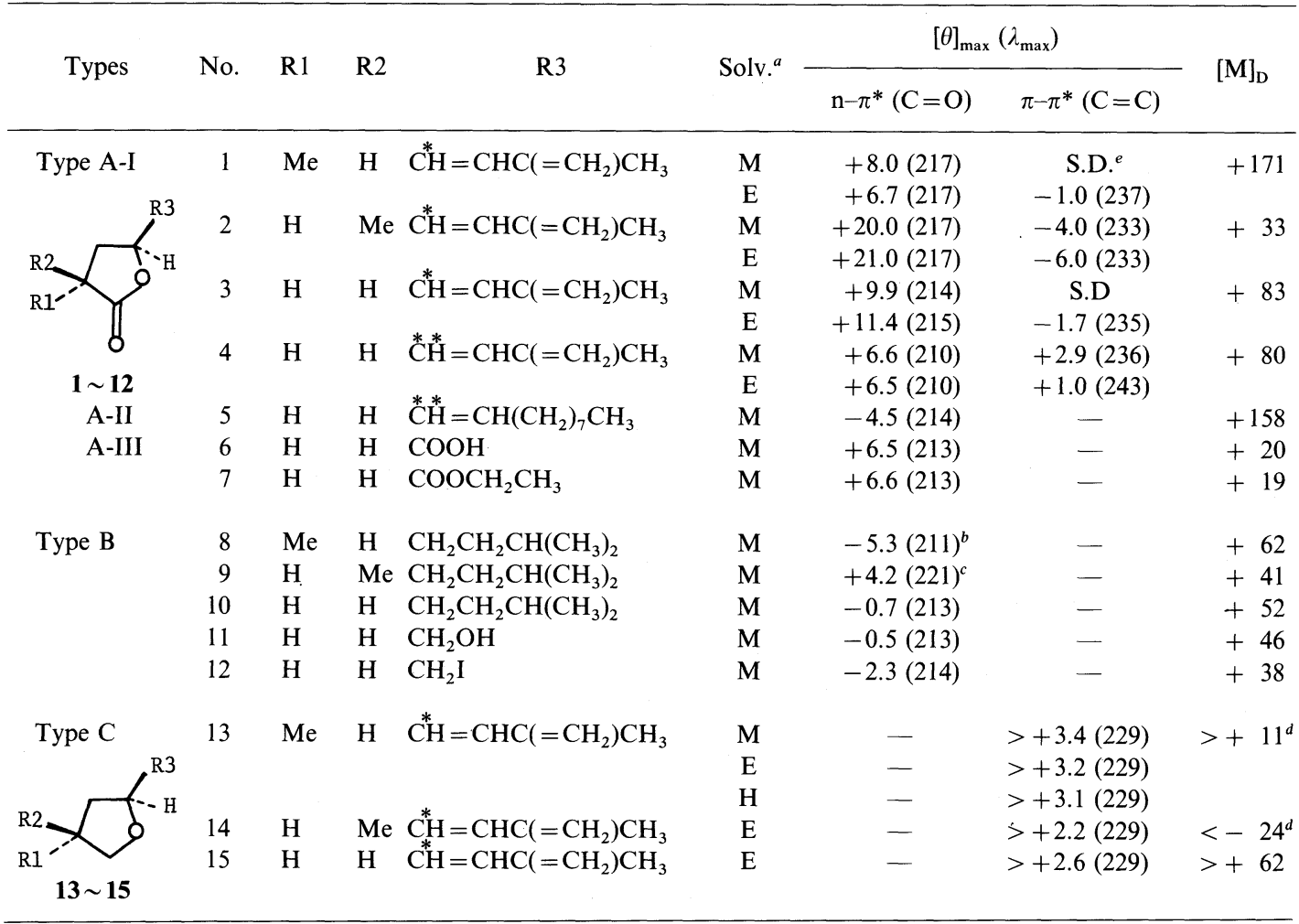

a Solvents: $\mathrm{M}=$ methanol; $\mathrm{E}=$ ethyl ether; $\mathrm{H}=n$-hexane.

$b, c$ Reference values. ${ }^{9)}$

${ }^{d}$ Optical purities have not yet been examined. About $70 \%$ e.e. could be expected from the $[\mathrm{M}]_{\mathrm{D}}$ values of the synthetic intermediates. ${ }^{6}$ )

e Shoulder bands.

* (5E)-Geometry.

** (5Z)-Geometry.

trostatic coupling or the dipole-dipole coupling between the $\mathrm{n} \rightarrow \pi^{*}$ transition of the carbonyl and the $\pi \longrightarrow \pi^{*}$ transition of the $\beta, \gamma-$ double bond. Judging from the clear couplet CD curves of the Type A-I lactones shown in Figs. 2 and 3, it seems clear that they also showed a coupling interaction between the two chromophores. This means that the (4S)lactones of Type A-I show a negative coupling which gives a negative $C D$ maximum in the $c a$. $235 \mathrm{~nm}$ region (the first band) and a positive maximum in the $c a .215 \mathrm{~nm}$ region (the second band). Here, the second band is very clear and the sign will have diagnostic value for the couplings, while the first band in the $c a$. $235 \mathrm{~nm}$ region is ambiguous and was signifi- cantly changed by the solvents (see Figs. 2 and 3), perhaps due to the overlap of another contribution in the same region.

Similar interaction was also expected for the intense CD band of the Type A-II lactones with a non-conjugated double bond. Since the UV maximum of the C-5 double bond shifted to a shorter wavelength region than the carbonyl $\mathrm{n} \rightarrow \pi^{*}$ transition (below $190 \mathrm{~nm}$ ), the negative CD band at ca. $215 \mathrm{~nm}$ of beetle's pheromone 5 indicates its negative coupling, similar to the negative coupling for the Type A-I lactones with a conjugated diene moiety.

The Type A-III lactones with a carbonyl group at the $\mathrm{C}-4$ position also show intense positive CD bands at $c a .213 \mathrm{~nm}$, similar to the 
Table II. $J_{\mathrm{H} 4, \mathrm{H} 5}$ Values of the C-5 Unsaturated $\gamma$-Lactones $1 \sim \mathbf{5}$, Oxolanes $13 \sim \mathbf{1 5}$, and the Related Acyclic Allylic Alcohols ${ }^{7,10}$ )

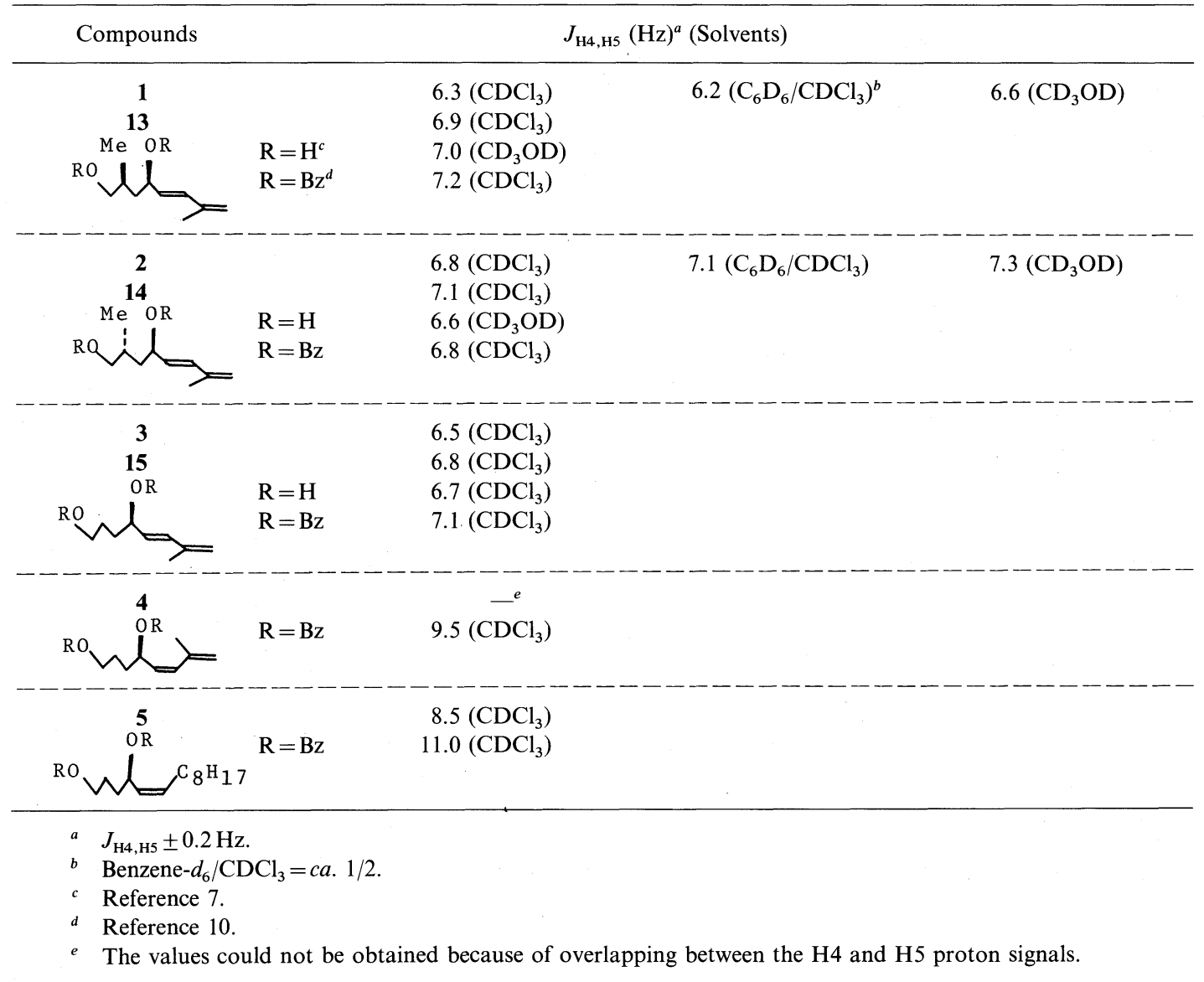

Type A-I lactones. Their CD signs will be useful for configurational analyses of the C-5 unsaturated lactones (Type A-I and Type AII), because the $-\mathrm{C}=\mathrm{C}-$ bond at $\mathrm{C}-5$ in the Type A-I and II lactones could be easily cleft into the carboxyl group by oxidative reactions with $\mathrm{O}_{3}$. However, the intensification of the $\mathrm{CD}$ band in the Type A-III lactones relative to the reduced lactones with a $\mathrm{CH}_{2} \mathrm{OH}$ group at $\mathrm{C}-4$ seems difficult to explain in a manner similar to that of the Type A-I and A-II lactones with a $\mathrm{C}=\mathrm{C}$ bond at the $\mathrm{C}-5$ position.

b) The preferred conformations about the C4$C 5$ bond in $\gamma$-lactones with a double bond at the $C-5$ position

In the preceding section, we found that the Type A-I and A-II lactones with a $\mathrm{C}=\mathrm{C}$ double bond at the $C-5$ position give a negative coupling interaction between the carbonyl and the C-5 double bond chromophores to reflect their (4S)-configuration. In the coupling mechanism, the absolute geometries of the two chromophores are important for determining the sign and magnitude of the produced bands. ${ }^{16)}$ Although the geometries may change due to free rotation about the $\mathrm{C} 4 \sim \mathrm{C} 5$ bond, their ${ }^{1} \mathrm{H}-\mathrm{NMR}$ data $\left(J_{\mathrm{H} 4, \mathrm{H} 5} \geq 6.2 \mathrm{~Hz}\right)$ shown in Table II indicate that the $\mathrm{C} 4-\mathrm{C} 5$ axis predominates in the conformation with the C-5 double bond in an eclipse relation with the C4-H4 bond (Fig. 4). This conformational property is known to be characteristic of the acyclic allylic alcohols ${ }^{11)}$ and was found to be kept in these unsaturated $\gamma$-lactones and oxolanes (Table II). 


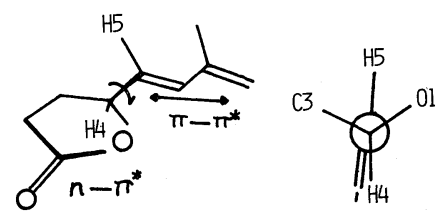

FIG. 4. Preferred Conformation about the C4-C5 Bond of C-5 Unsaturated $\gamma$-Lactones.

Here, it should be noticed that the lactones 4 and 5 take almost the same conformation about the $\mathrm{C} 4 \mathrm{C} 5$ axis, judging from their NMR data, giving positive and negative CD maxima in the $c a .215 \mathrm{~nm}$ region, respectively, as shown in Fig. 3, irrespective of the same absolute geometries between the two chromophores. The CD changes between 4 and 5 could not be expected from the 'lactone sector' rule, ${ }^{1,2)}$ but are well explained with the coupling mechanism between the two chromophores as already mentioned. Moreover, these data strongly suggest that the conformational property about the $\mathrm{C} 4-\mathrm{C} 5$ axis in the Type A-I and A-II lactones would be an important factor for their negative interaction, although the mechanism has not yet been theoretically clarified.

In conclusion, we found that $\gamma$-lactones with unsaturation at the $\mathrm{C}-5$ position give an intense $C D$ band in the $c a .215 \mathrm{~nm}$ region to reflect their $\mathrm{C}-4$ configurations. The intensification can be well explained by coupling interactions betwen the $\mathrm{n} \rightarrow \pi^{*}$ transition of the carbonyl and the $\pi \rightarrow \pi^{*}$ transition of the C-5 double bond, similar to that of the $\beta, \gamma$-unsaturated ketones. The $(4 S)-\gamma$-lactones showed a negative coupling to which the intense positive band of (4S)-marmelo lactones and the negative band of ( $4 S$ )-Japanese Beetle's sex pheromone could be attributed. These results will become important bases for spectrometri- cal studies on the $\gamma$-lactones, and for their configurational and conformational analyses, together with the other method ${ }^{10)}$ based on the benzoate chirality rule. ${ }^{16)}$

Acknowledgments. We are most grateful to Professor Toshio Konno of Niigata University for his useful advice during this study. This work was supported in part by a Grant-in-Aid for Scientific Research from the Ministry of Education, Science and Culture of Japan.

\section{REFERENCES}

1) J. P. Jennings, W. Klyne and P. M. Scopes, J. Chem. Soc., 7211 (1965).

2) G. Snatzke, H. Ripperger, C. Horstmann and K. Schreiber, Tetrahedron, 22, 3103 (1966).

3) T. Okuda, S. Harigaya and A. Kiyomoto, Chem. Pharm. Bull. Jpn., 12, 504 (1964).

4) A. F. Beecham, Tetrahedron Lett., 2355 (1968).

5) H. Meguro, T. Konno and K. Tuzimura, Tetrahedron Lett., 1305 (1975); ibid., 1309 (1975).

6) Y. Nishida, H. Ohrui and H. Meguro, Agric. Biol. Chem., 48, 1211 (1984).

7) Y. Nishida, Y. Fukushima, H. Ohrui and H. Meguro, Agric. Biol. Chem., 48, 1217 (1984).

8) Y. Nishida, T. Konno, H. Ohrui and H. Meguro, Agric. Biol. Chem., 47, 2683 (1983).

9) M. Ishihara, T. Tsuneya, H. Shiota, M. Shiga and Y. Yokoyama, Agric. Biol. Chem., 47, 2121 (1983).

10) Y. Nishida, M. Konno, H. Ohrui and H. Meguro, Agric. Biol. Chem., 50, 187 (1986).

11) N. C. Gonnella, K. Nakanishi, U. S. Martin and K. B. Sharpless, J. Am. Chem. Soc., 104, 3775 (1982).

12) S. Senda and K. Mori, Agric. Biol. Chem., 47, 2595 (1983).

13) A. Moscowitz, E. Charney, U. Weiss and H. Ziffer, $J$. Am. Chem. Soc., 83, 4661 (1961).

14) A. Moscowitz, A. E. Hansen, L. S. Forster and K. Rosenheck, Biopolym. Symp., 1, 75 (1964).

15) S. Hagishita and K. Kuriyama, J. Chem. Soc., Perkin Trans. 2, 1937 (1977).

16) N. Harada and K. Nakanishi, "Circular Dichroic Spectroscopy Exciton Coupling in Organic Stereochemistry," University Science Books, California, U.S.A., 1983. 